\title{
Blood-Brain Barrier Active Efflux Transporters: ATP-Binding Cassette Gene Family
}

\author{
Wolfgang Löscher and Heidrun Potschka \\ Department of Pharmacology, Toxicology, and Pharmacy, University of Veterinary Medicine Hannover, \\ Hannover D-30559, Germany
}

\begin{abstract}
Summary: The blood-brain barrier (BBB) contributes to brain homeostasis by protecting the brain from potentially harmful endogenous and exogenous substances. BBB active drug efflux transporters of the ATP-binding cassette $(\mathrm{ABC})$ gene family are increasingly recognized as important determinants of drug distribution to, and elimination from, the CNS. The ABC efflux transporter P-glycoprotein (Pgp) has been demonstrated as a key element of the BBB that can actively transport a huge variety of lipophilic drugs out of the brain capillary endothelial cells that form the BBB. In addition to Pgp, other ABC efflux transporters such as members of the multidrug resistance protein (MRP) family and breast cancer resistance protein (BCRP) seem to contribute to $\mathrm{BBB}$ function. Consequences of $\mathrm{ABC}$
\end{abstract}

efflux transporters in the BBB include minimizing or avoiding neurotoxic adverse effects of drugs that otherwise would penetrate into the brain. However, ABC efflux transporters may also limit the central distribution of drugs that are beneficial to treat CNS diseases. Furthermore, neurological disorders such as epilepsy may be associated with overexpression of $A B C$ efflux transporters at the $\mathrm{BBB}$, resulting in pharmacoresistance to therapeutic medication. Therefore, modulation of $\mathrm{ABC}$ efflux transporters at the BBB forms a novel strategy to enhance the penetration of drugs into the brain and may yield new therapeutic options for drug-resistant CNS diseases. Key Words: P-glycoprotein, multidrug resistance proteins, epilepsy, antiepileptic drugs, depression, AIDS.

\section{INTRODUCTION}

ATP-binding cassette $(\mathrm{ABC})$ transporters are multidomain integral membrane proteins that use the energy of ATP hydrolysis to translocate solutes across cellular membranes in all mammalian species. ${ }^{1} \mathrm{ABC}$ transporters form one of the largest of all protein families and are central to many important biomedical phenomena, including resistance of cancers and pathogenic microbes to drugs. $^{2}$ Elucidation of the structure and function of $\mathrm{ABC}$ transporters is essential to the rational design of agents to control their function.

$\mathrm{ABC}$ transporters are increasingly recognized to be important for drug disposition and response. ${ }^{3-7} \mathrm{P}$-glycoprotein (Pgp), the encoded product of the human multidrug resistance (MDRl) (ABCB1) gene, is of particular clinical relevance in that this transporter has a broad substrate specificity, including a variety of structurally divergent drugs in clinical use today. ${ }^{7-9}$ Moreover, ex-

Address correspondence and reprint requests to Dr. W. Löscher, Department of Pharmacology, Toxicology and Pharmacy, University of Veterinary Medicine Hannover, Foundation, Bünteweg 17, D-30559 Hannover, Germany. E-mail: wolfgang.loescher@tiho-hannover.de. pression of this efflux transporter in certain tissue compartments such as the gastrointestinal tract and brain capillary endothelial cells limits oral absorption and CNS entry of many drugs. ${ }^{7}$ The use of Pgp-expressing cell lines, the generation of Pgp knockout mice as well as studies using Pgp inhibitors in animals, contributed to a better understanding on the role of active transport processes for drug disposition. ${ }^{8}$ In addition to Pgp, the ABC transporters of the multidrug resistance protein (MRP; $\mathrm{ABCC}$ ) family and the breast cancer resistance protein (BCRP; ABCG2) have a role in drug disposition. ${ }^{6,9}$ The family of mammalian $\mathrm{ABC}$ transporters, however, is far more extensive, and functionally highly diverse. ${ }^{1}$ In this review, we limit ourselves to the following $\mathrm{ABC}$ transporters: Pgp, MRPs 1-6, and BCRP, i.e., ABC transporters that are expressed at the blood-brain barrier (BBB) and, particularly Pgp, are involved in the regulation of brain uptake and extrusion of drugs. ${ }^{6,7,9,10}$

\section{WHICH ABC TRANSPORTERS ARE EXPRESSED AT THE BBB?}

Drug uptake into the brain is dependent on a variety of factors, including the physical barrier presented by the 
TABLE 1. Substrates and Inhibitors of ABC Transporters that Are Expressed at the Blood-Brain Barrier

\section{$\mathrm{ABC}$}

Transporter

P-glycoprotein

(ABCB1)

MRP1 (ABCC1)

MRP2 (ABCC2)

MRP3 (ABCC3)

MRP4 (ABCC4)

MRP5 (ABCC5)

MRP6 (ABCC6)

BCRP (ABCG2)
Substrates

Inhibitors
Anticancer drugs e.g., Doxorubicine, daunorubicine, vinblastine, vincristine, etoposide, teniposide, paclitaxel, methotrexate

Immunosuppressive agents e.g., Cyclosporin A

Corticoids e.g., Dexamethasone, hydrocortisone, corticosterone, cortisol, aldosterone

Analgesics e.g., Morphine

HIV protease inhibitors e.g., Amprenavir, indinavir, saquinavir

Cytokines e.g., IL-2, IL-4, IFN- $\gamma$

Antidiarrheal agents e.g., Loperamide

Anthelminthic agents e.g., Ivermectin, abamectin

Anti-gout agents e.g., Colchicines

Histamine H2-receptor antagonists e.g., Cimetidine

Calcium channel blocker e.g., verapamil

Antiepileptic drugs e.g., Phenytoin, carbamazepine, lamotrigine, phenobarbital, felbamate, gabapentin, topiramate

Antiemetics e.g., Domperidone, ondansetron

Cardiac glycosides e.g., Digoxin

Diagnostic (fluorescent) dyes e.g., Rhodamine-123

Antidepressants e.g., Amitryptiline, nortryptiline, doxepin, venlafaxine, paroxetine

Antibiotics e.g., Erythromycin, valinomycin, tetracyclines, fluoroquinolines

Anticancer drugs e.g., Etoposide, teniposide, vincristine, doxorubicine, daunorubicine, methotrexate; Leukotriene C4 (LTC4), D4, E4; Various glutathione, glucuronide, and sulfate conjugates, but also unconjugated compounds (e.g., fluorescein)

Similar to MRP1

Organic anion transporter with considerable overlap in drug substrates with MRP1 and MRP2

Anticancer drugs such as methotrexate, 6-mercaptopurine, thioguanine

cGMP, cAMP, 6-mercaptopurine, thioguanine, fluorescein

BQ-123 (an anionic cyclopentapeptide and endothelin receptor antagonist)

Several anticancer drugs; considerable overlap with Pgp, MRP1, and MRP2. Anthracyclines, mitoxantrone, bisantrene, the camptothecins topotecan and SN-38, prazosin 1st Generation e.g., verapamil, cyclosporin A, quinidine, quinine, amiodarone, detergents such as cremophore EL

2nd Generation e.g., PSC-833 (valspodar), GF120918 (elacridar), VX-710 (biricodar), dexverapamil

3rd Generation e.g., OC 144-093 (ONT093), LY335979 (zosuquidar), XR9576 (tariquidar), R101933 (laniquidar), GF120918

Sulfinpyrazone, probenecid, MK571, LTC4, some Pgp inhibitors (e.g., cyclosporin A, verapamil, PSC 833)

Similar to MRP1

Classical organic anion transport inhibitors such as sulfinpyrazone, indomethacin, and probenecid

Probenecid and phosphodiesterase inhibitors such as trequensin or sildenafil

GF120918 (inhibits also Pgp), fumitremorgin C (FTC) and FTC analogues such as Ko132 and Ko134, CI1033

For reference see Schinkel and Jonker, ${ }^{6}$ Sun et al., ${ }^{9}$ Gerk and Vore, ${ }^{11}$ and Fricker and Miller. ${ }^{14}$

BBB and the blood-CSF barrier (BCSFB) and the affinity of the substrate for specific transport systems located at both of these interfaces. ${ }^{11,12}$ It is the aggregate effect of these factors that ultimately determines the total brain exposure, and thus pharmacological efficacy, of a drug or drug candidate. In general, the more lipid soluble a molecule or drug is, the more readily it will tend to partition into brain tissue. However, a very significant number of lipid-soluble molecules, among them many useful therapeutic drugs have lower brain permeability than would be predicted from a determination of their lipid solubility. These molecules are substrates for the ABC efflux transporters (Table 1) that are present in the BBB and $\mathrm{BCSFB}$, and the activity of these transporters very effi- 
ciently removes the drug from the CNS, thus limiting brain uptake.

Pgp, a phosphorylated glycoprotein with an apparent molecular mass of $170 \mathrm{kDa}$, was the first of these ABC transporters to be described, followed by the MRPs and more recently BCRP. ${ }^{9}$ All are expressed in the BBB or BCSFB and combine to reduce the brain penetration of many drugs. This phenomenon of multidrug resistance is a major hurdle when it comes to the delivery of therapeutics to the brain, including brain cancer chemotherapy. Therefore, the development of strategies for bypassing the influence of these $\mathrm{ABC}$ transporters, for the design of effective drugs that are not substrates, and for the development of inhibitors for the $\mathrm{ABC}$ transporters has become a high imperative for the pharmaceutical industry. ${ }^{15}$

The BBB is a physical and metabolic barrier between the brain and the systemic circulation, which serves to regulate and protect the microenvironment of the brain. ${ }^{11}$ The BBB is composed of a monolayer of brain capillary endothelial cells. The restriction of brain uptake by the $\mathrm{BBB}$ arises by the presence of tight junctions (zonulae occludens) between adjacent endothelial cells and a relative paucity of fenestrae and pinocytotic vesicles within endothelium of cerebral arterioles, capillaries, and venules. The brain capillary endothelial cells are surrounded by an ECM, pericytes, and astrocyte foot processes. Because of the presence of the BBB, circulating molecules gain access to the brain only via one of two processes: 1) lipid-mediated transport of small nonpolar molecules through the BBB by free (passive) diffusion, or 2) catalyzed transport. ${ }^{11}$ The endothelial cells of the BBB contain numerous membrane transporters involved in the influx or efflux of various essential substrates such as electrolytes, nucleosides, amino acids, and glucose. ${ }^{16}$ It was originally believed that membrane carriers localized at the BBB were solely responsible for the transport of such endogenous substances into and out of the brain and that drug transport across the BBB was largely dependent on the physicochemical characteristics of the drug such as lipophilicity, molecular weight, and ionic state. However, ABC efflux transporters at the BBB limit the brain uptake of a variety of therapeutic agents, including compounds that are relatively lipophilic and would be predicted to permeate the endothelial lining of the brain microvasculature. ${ }^{17}$

Initially discovered in the 1970 s as a prototypic transporter involved in multidrug resistance (MDR) of cancer cells, ${ }^{18}$ Pgp was also the first ABC transporter that was detected in endothelial cells of the human BBB in 1989. ${ }^{19,20}$ Subsequently Pgp was localized in brain capillary endothelial cells of several species, including monkeys, rats, mice, cattle, and pigs, suggesting that Pgp may serve as general defense mechanism in the mammalian $\mathrm{BBB}$, protecting the brain from intoxication by poten- tially harmful lipophilic compounds from natural sources and other lipophilic xenobiotics which otherwise could penetrate the BBB by simple diffusion without any limitation. ${ }^{10}$ The exact localization of Pgp in the BBB has been the object of numerous investigations using various experimental approaches such as in situ hybridization and immunohistochemistry. ${ }^{21}$ There are two types of human Pgp: type I (also termed "MDR1 Pgp"), encoded by the MDRl gene, which confers the drug resistance phenotype and drug efflux at the BBB (only this type will dealt with in this review); and type II, encoded by MDR2, present in the canalicular membrane of hepatocytes and functioning as a phosphatidylcholine translocase. ${ }^{21}$ In rodents, the multidrug resistance type I Pgp is encoded by two genes $(m d r l a, m d r l b){ }^{21}$ The substrate specificity of mdrla and $m d r l b$ Pgp in the rodent is different but partly overlapping, and together the two rodent genes are expressed in roughly the same manner as the single human MDR1 gene, suggesting that they perform the same set of functions in rodents as the MDR1 Pgp in man. ${ }^{10}$ Both $m d r l a$ and $m d r l b$ are present in rodent brain, but only mdrla is localized in brain capillaries of mice and rats, whereas mdrlb is present only in brain parenchyma. ${ }^{21}$ At the subcellular level, most of the published data demonstrate that Pgp [or the gene(s) encoding Pgp] are principally expressed at the luminal (apical) membrane of brain capillary endothelial cells in mammals, including humans (FIG. 1). ${ }^{6,9,21}$ Thus, Pgp substrate drugs entering the endothelial cells from the blood are immediately pumped back into the blood. As a consequence, the net penetration of substrate drugs and other substrate compounds from the blood into the brain tissue can be dramatically decreased. ${ }^{10}$ In the absence of Pgp in the BBB in mdrla knockout mice, the brain penetration of Pgp substrate drugs can increase up to 10 - to 100 -fold, with sometimes dramatic consequences for the toxicity of compounds. ${ }^{6}$ Furthermore, blockade of BBB Pgp by cerebral application of Pgp inhibitors significantly increases the brain concentration of various drugs, again being in line with Pgp functioning as an efflux transporter in the BBB..$^{22,23}$

However, there has been some controversy regarding the localization of Pgp in the BBB. Studies by Pardridge et al., ${ }^{24}$ using the mouse MRK16 monoclonal antibody to human Pgp, suggested that the pattern of immunolocalization of Pgp in human and primate brain was the same as that of glial fribrillary acidic protein (GFAP), a protein found in astrocytes. Moreover, in this study the authors found that Pgp did not colocalize with the brain endothelial GLUT1 glucose transporter. Similar findings were reported using unfixed isolated human brain capillaries. ${ }^{25}$ These findings led this group to hypothesize a revised model of multidrug resistance in the brain, which places the Pgp transporter predominantly at the astrocyte foot processes ensheathing brain microvessels rather 


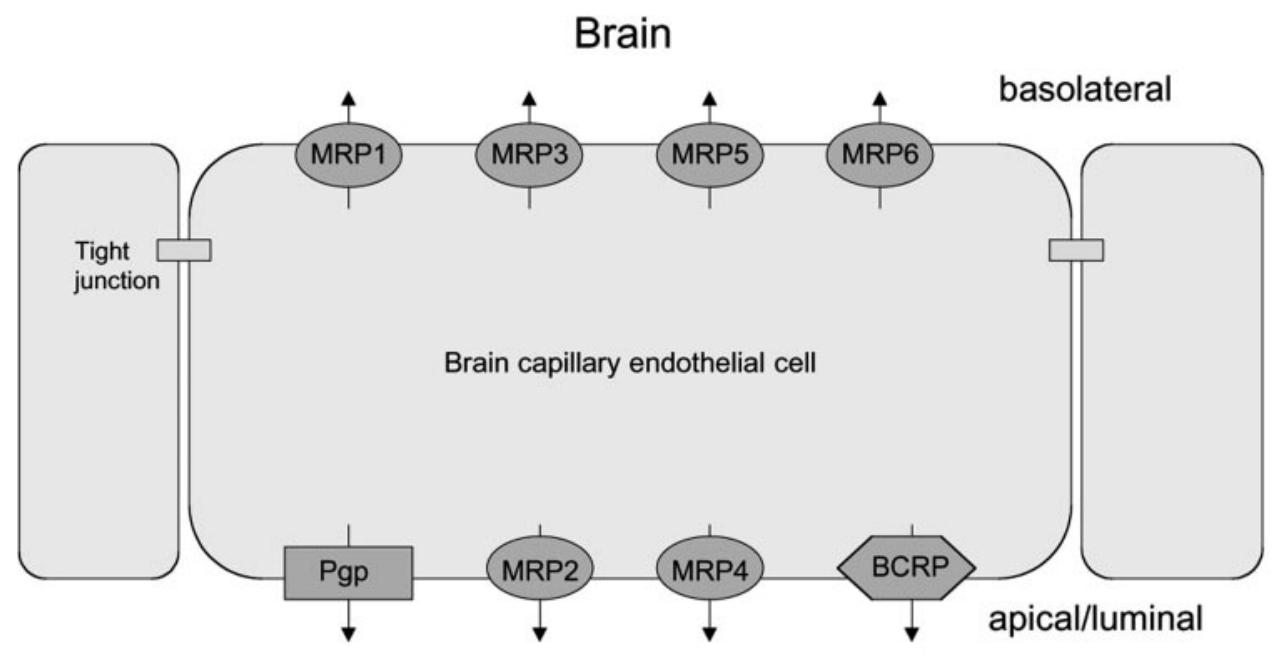

Blood

FIG. 1. Localization of selected drug efflux proteins on brain capillary endothelial cells that form the blood-brain barrier. Only transporters that are localized on the apical (luminal) side of the brain capillary endothelium would be in a position to restrict brain uptake of xenobiotics. Note that the exact localization in endothelial cells has not been demonstrated as yet for all transporters shown in the figure, but for some of the transporters the localization (apical vs basolateral) was derived from studies on polarized epithelial cell lines. ${ }^{6}$

than the endothelial luminal membrane. ${ }^{26}$ By this model, the increased brain-to-plasma ratio of Pgp substrates, observed in both mdrla knockout mice and animals treated with Pgp inhibitors, could be due to increased intracellular concentrations in astrocytes that express Pgp, thereby resulting in an increased brain volume of distribution of Pgp substrates. However, this model would not explain that Pgp inhibitors increase extracellular brain drug levels as determined by microdialysis in rodents. ${ }^{23}$ The conclusions of Pardridge and colleagues have been criticized ${ }^{10,27}$ because they were essentially based on findings with only one Pgp antibody (MRK16). It is known that the extracellular epitope of human Pgp for the monoclonal antibody MRK16 can be completely shielded in some cell types by heavy $\mathrm{N}$-glycosylation. ${ }^{10}$ Pgp immunohistochemistry is further highly sensitive to preparation and fixation of samples. Suboptimal fixation may lead to lack of recognition by obliteration of epitopes, or increased aspecific binding of the primary antibody. ${ }^{10,28}$ Based on these complications, immunolocalizations of Pgp can only be considered reliable when consistent results are obtained with at least two, and preferably three or more different antibodies. ${ }^{10,29}$ In a recent study, using a rabbit polyclonal antiserum [mdr(Ab-1)] against Pgp and different tissue fixation protocols, Schlachetzki and Pardridge ${ }^{30}$ demonstrated a dual expression of Pgp at both astrocytes and endothelium of normal primate brain, thus obviously resolving the previous conflicting results. However, still most other groups did not detect Pgp in astrocytes of normal brain tissue, indicating that fixation and staining variables may be critical. Clear expression of Pgp in astrocytes is seen in certain pathological states such as epilepsy. ${ }^{31,32}$ By using three different Pgp antibodies to localize Pgp in rat brain, we have recently found that tissue fixation and staining conditions have a striking effect on the immunohistochemical localization of Pgp in rat brain and detection of its increased expression by seizures. ${ }^{32 a}$ Our experiments demonstrated that, under physiological conditions, Pgp is predominantly expressed by capillary endothelial cells but, although much weaker, also by parenchymal and perivascular astrocytes, which is in line with the recent study of Schlachetzki and Pardridge ${ }^{30}$ in primate brain. Seizures induced an overexpression of Pgp in these different cell types and, in addition, lead to Pgp expression in neurons. ${ }^{32 a, 33}$ The expression of Pgp in these four different cell types (i.e., brain capillary endothelial cells, perivascular astrocytes, parenchymal astrocytes, and neurons) suggests that Pgp's functions in the brain may be more complex than previously thought.

In contrast to Pgp, data on other $\mathrm{ABC}$ transporters in the BBB are much more limited. ${ }^{6,9}$ The ABCC family (with the first member, MRP1, discovered in cancer cells in 1992) currently has 12 members (including MRP1-9) (see www.gene.ucl.ac.uk/nomenclature/genefamily/abc), which act as organic anion transporters, but can also transport neutral organic drugs. ${ }^{34}$ As a consequence, PGP and MRPs have overlapping substrate specificity, so that several drugs are substrates for both transporter families. ${ }^{6,34,35}$ Like PGP, MRPs are located in several normal tissues, including the BBB and BCSFB. ${ }^{34}$ Some MRPs, like MRP2, are located in apical cell membranes of tissues, which in most membranes is the appropriate position for a cytoprotective role by an efflux transporter, 
whereas other MRPs, such as MRP1, MRP3, and MRP5, are located basolaterally. ${ }^{34}$ Expression of MRPs in brain microvessel endothelial cells that form the BBB has been reported for several species, including humans. ${ }^{15}$ Using primary cultured bovine brain microvessel endothelial cells and the capillary-enriched fraction from bovine brain homogenates, RT-PCR analysis demonstrated the presence of mRNAs coding for MRP1, MRP4, MRP5, and MRP6 as well as low levels of MRP3 mRNA, whereas MRP2 mRNA was absent. ${ }^{37}$ However, using immunostaining of PGP and MRP2 in isolated capillaries from rat and pig brain, both multidrug transporters were localized to the luminal surface of the capillary endothelium. ${ }^{38}$ Using cDNA arrays to determine MRP expression in brain capillary endothelial cells isolated from humans, high expression levels of mRNAs coding for MRP1 and low expression levels of mRNAs coding for MRP2, MRP3, and MRP5 were reported by Dombrowski et al. ${ }^{39}$ Thus, taken together, at least six MRPs are expressed at the BBB of different species. However, the exact subcellular localization (apical $v s$ basolateral) of most of these MRPs in brain capillary endothelial cells remains to be determined, particularly because of the lack of selective antibodies. Furthermore, the expression level of MRP2 at the BBB is still in question. Assuming that only some MRPs are located at the luminal (apical) plasma membrane of brain capillary endothelial cells as illustrated in Fig 1, only these MRPs would be relevant in restricting brain access of MRP substrates.

In the rat brain, MRP1 is present in higher levels in astrocytes than in brain capillary endothelial cells. ${ }^{40}$ Furthermore, high expression of MRP1 is found in choroid plexus epithelial cells that form the BCSFB. ${ }^{41}$ In addition to MRP1, MRP4, and MRP5 mRNAs were expressed in the choroid plexus of rats at higher levels than in liver, kidney, or intestine, whereas MRP2 and MRP3 (as well as mdrla and $m d r l b$ ) were expressed only at very low levels at the BCSFB. ${ }^{42}$ Apart from the BBB and BCSFB, several MRPs have also been determined in astrocytes and microglial cells. ${ }^{43}$ The recent generation of mrp gene knockout mice is providing information on the physiological functions of MRPs in these different localizations. Mice lacking an intact mrpl gene have an altered response to inflammatory stimuli and show an increased toxic response to the anticancer drug etoposide but are otherwise healthy. ${ }^{34}$ The brain penetration of the MRP1 substrate fluorescein is not increased in mrp1 knockout mice, ${ }^{44}$ indicating that MRP1 function in the BBB is limited. In mdrla/mdrlb/mrpl triple knockout mice, but not in $m d r l a / m d r l b$ double knockout mice, etoposide levels in the CSF are markedly increased, indicating that MRP1 critically contributes to the permeability of the BCSFB. ${ }^{45}$ In addition to mrp knockout mouse mutants, there is a mrp2-deficient rat mutant $\left(\mathrm{TR}^{-}\right)$that can be used to study physiological functions of MRP2. ${ }^{46}$ The role of MRPs in BBB permeability has been demonstrated by experiments in which inhibitors of MRPs, such as probenecid or MK-571, were shown to enhance drug penetration into the brain or to inhibit drug efflux from isolated brain endothelial cells. ${ }^{23,44,47-49}$ Furthermore, in $\mathrm{TR}^{-}$rats, brain extracellular levels of the antiepileptic drug (AED) phenytoin are significantly enhanced compared with normal rats (whereas plasma drug levels are the same), indicating that MRP2 expression at the $\mathrm{BBB}$ restricts brain entry of this compound. ${ }^{50}$

BCRP was first discovered in a chemotherapy-resistant breast cancer cell line, but there is no indication that its expression is specific for breast cancer cells or that BCRP should play a significant role in chemotherapyresistance in breast cancer. ${ }^{6}$ The tissue distribution of BCRP shows extensive overlap with that of Pgp, suggesting that both transporters similarly confer protection from potentially harmful xenobiotics in various tissues. ${ }^{6}$ In the brain, BCRP has been detected in capillary endothelial cells of pigs, ${ }^{51}$ mice, ${ }^{52}$ and humans, mainly at the luminal surface. ${ }^{53}$ Based on mRNA analysis, BCRP was more strongly expressed at the $\mathrm{BBB}$ than Pgp or MRP1. ${ }^{51}$ The role of BCRP in brain uptake was recently demonstrated by using mdrla knockout mice, in which BCRP was inhibited by GF120918, resulting in reduced brain uptake of prazosin and mitoxantrone. ${ }^{52}$ Interestingly, mdrla knockout mice had about three times more BCRP in the brain microvessels than normal mice, indicating an upregulation of BCRP to compensate for the lack of Pgp in the BBB. ${ }^{52}$

A list of representative substrates of Pgp, MRPs, and BCRP is shown in Table 1 together with inhibitors of these multidrug transporters. The list of compounds shown in this table is not meant to be all inclusive, but rather provides an appreciation for the chemical diversity of compounds that are recognized as substrates by these ABC transporters. Furthermore, as shown in this table, there is considerable overlap between substrates for these efflux transporters. With respect to the multiple binding sites and complex mechanisms of substrate recognition and transport, the interested reader is referred to several previous papers on this topic. ${ }^{35,36,54-57}$ However, it is still not clear how ABC transporters recognize and translocate substrates. ${ }^{27}$

Weak substrates of BBB efflux transporters generally pass the BBB to a certain extent, and exert CNS effects. However, their effectiveness is critically influenced by expression rates and the functional state of the relevant transporter, which influences brain uptake and extrusion rates. ${ }^{9}$ In contrast, strong substrates of BBB efflux transporters do not pass the $\mathrm{BBB}$ to a functionally relevant extent, and their pharmacodynamic effects are restricted to the periphery. ${ }^{6}$ Affinity of these drugs to the respective efflux transporter seems to be that high that the vast majority of the drug gets into contact with the transporter 
TABLE 2. Overexpression of ABC Transporters in Epileptogenic Brain Tissue

\begin{tabular}{|c|c|c|c|c|c|}
\hline \multirow[b]{2}{*}{$\begin{array}{l}\text { ABC Transporter } \\
\text { (Protein and/or Gene) }\end{array}$} & \multirow[b]{2}{*}{$\begin{array}{l}\text { Epileptogenic Brain } \\
\text { Tissue from }\end{array}$} & \multicolumn{3}{|c|}{$\mathrm{ABC}$ Transporter Overexpressed in } & \multirow[b]{2}{*}{ Reference } \\
\hline & & $\begin{array}{c}\text { Capillary } \\
\text { EndothelialCells }\end{array}$ & Astrocytes & Neurons & \\
\hline \multirow[t]{4}{*}{ P-glycoprotein $(M D R I)$} & Patients & + & + & + & $\begin{array}{l}\text { Tishler et al., }{ }^{63} \text { Sisodiya et al., }{ }^{65,66} \\
\text { Dombrowski et al., }{ }^{39} \text { Aronica et } \\
\text { al.67,68 }\end{array}$ \\
\hline & Rats (kainate) & + & + & + & $\begin{array}{l}\text { Zhang et al., }{ }^{69} \text { Seegers et al., }{ }^{70} \\
\text { Volk et al., }{ }^{33,71}\end{array}$ \\
\hline & Rats (pilocarpine) & $?$ & + & + & Volk et al. \\
\hline & Rats (kindling) & + & ? & $?$ & Volk et al. ${ }^{71}$ \\
\hline MRP1 & Patients & - & + & + & $\begin{array}{l}\text { Sisodiya et al.,.65,72 Dombrowski et } \\
\text { al., }{ }^{39} \text { Aronica et al. }{ }^{67}\end{array}$ \\
\hline MRP2 & Patients & + & + & $?$ & $\begin{array}{l}\text { Dombrowski et al., }{ }^{39} \text { Aronica et } \\
\text { al. }{ }^{68}\end{array}$ \\
\hline MRP3 & Patients & - & $?$ & $?$ & Dombrowski et al. ${ }^{39}$ \\
\hline MRP5 & Patients & + & $?$ & $?$ & Dombrowski et al. ${ }^{39}$ \\
\hline BCRP & Patients & - & - & - & Sisodiya et al. ${ }^{73}$ \\
\hline
\end{tabular}

immediately after entering the endothelial cell membrane or cytoplasm and is directly extruded into the capillary lumen.

\section{ROLE OF THE BBB ABC TRANSPORTERS IN DRUG RESISTANCE}

The phenomenon of multidrug resistance has been acknowledged for many years as a major obstacle in cancer therapies and is characterized by resistance to a broad range of structurally and functionally unrelated cytotoxic agents. ${ }^{23}$ However, multidrug resistance also occurs in other diseases such as rheumatoid arthritis, epilepsy, or certain psychiatric diseases, and interest in multidrug transporters such as Pgp in the mechanisms underlying drug resistance in such diseases is currently receiving much attention. ${ }^{23,32,58,59}$ In brain diseases such as epilepsy, depression, or schizophrenia about $20-40 \%$ of patients are resistant to current medications despite adequate choice of therapeutic drugs at maximum tolerated doses, so that new therapeutic options for such patients are urgently needed. ${ }^{60-62}$

Tishler et al. ${ }^{63}$ were the first to report that brain expression of MDRl, which encodes the multidrug transporter PGP in humans, is markedly increased in the majority of patients with medically intractable partial (mostly temporal lobe) epilepsy. MDR1 mRNA levels were determined by RT-PCR in brain specimens removed from patients during resective surgery for intractable epilepsy and were compared with normal brain control specimens obtained from patients undergoing removal of arteriovenous malformations. In line with enhanced MDR1 expression in epileptogenic brain tissue, immunohistochemistry for Pgp showed increased staining in capillary endothelium and astrocytes. Tishler et al. ${ }^{63}$ proposed that Pgp may play a clinically significant role by limiting access of AEDs to the brain parenchyma, so that increased MDR1 expression may contribute to the refractoriness of seizures in patients with treatment-resistant epilepsy. Subsequently, it was shown by other groups that, in addition to PGP, several MRPs, but not BCRP are overexpressed in capillary endothelial cells and/or astrocytes of pharmacoresistant patients (Table 2). In some of these studies, the overexpression in astrocytes appeared most marked around blood vessels. In view of data indicating that the endothelial barrier function of the $\mathrm{BBB}$ is transiently disrupted during seizures,${ }^{64}$ overexpression of multidrug transporters in glial end-feet covering the blood vessels may represent a second barrier under these conditions. ${ }^{65}$ Sisodiya et al. ${ }^{65}$ proposed that overexpressed multidrug transporters lower the extracellular concentration of AEDs in the vicinity of the epileptogenic pathology and thereby render the epilepsy caused by these pathologies resistant to AED treatment.

An open question is whether the overexpression of PGP and MRPs in epileptogenic brain tissue of patients with intractable epilepsy is intrinsic (constitutive) or acquired, i.e., a consequence of epilepsy, of uncontrolled seizures, of chronic treatment with AEDs, or of combinations of these factors. Because treatment-resistant patients have the same extent of neurotoxic side effects under AED treatment as patients who are controlled by AEDs, the overexpression of drug transporters in treatment-resistant patients is most likely restricted to the epileptic focus or circuit. This is substantiated by a previous study of Sisodiya et al. ${ }^{65}$ in which overexpression of PGP and MRP1 was found in epileptogenic tissue but not adjacent normal tissue of the same patients.

In animal models of temporal lobe epilepsy (TLE), seizures have been found to transiently overexpress PGP 
in brain capillary endothelial cells, astroglia, and neurons (Table 2), indicating that seizures rather than epilepsy are responsible for overexpression of drug transporters. This could explain that one of the major predictors of pharmacoresistance is high seizure frequency before initiation of treatment. ${ }^{74}$ However, constitutive rather than induced or acquired overexpression of multidrug transporters has been reported in patients with malformations of cortical development. ${ }^{66}$ In addition to intrinsic or acquired overexpression of multidrug transporters in the $\mathrm{BBB}$ of patients with epilepsy, functional polymorphisms of these transporters may play a role in pharmacoresistance. ${ }^{75,76}$ Furthermore, both overexpression and functional polymorphisms of multidrug transporters in patients with intractable epilepsy need not necessarily be restricted to the brain, but could also occur in other tissues, such as the small intestine, where PGP is thought to form a barrier against entrance of drugs from the interstinal lumen into the bloodstream, thereby limiting their oral bioavailability. ${ }^{4}$ In this respect, it is interesting to note that Lazarowski et al. ${ }^{77}$ have reported persistent subtherapeutic plasma levels of AEDs (including phenytoin and phenobarbital) despite aggressive and continuous AED administration in a patient with refractory epilepsy associated with overexpression of MDRI.

In view of the emerging evidence that multidrug transporters are overexpressed in epileptogenic brain tissue, particularly in capillary endothelial cells and astrocytes contributing to BBB permeability, it is of major clinical interest to evaluate whether AEDs are substrates for these transporters. Only then, overexpression of PGP or MRPs could critically contribute to pharmacoresistance in epilepsy. By using a rat microdialysis model with microdialysis probes in both brain hemispheres and local (cerebral) inhibition of multidrug transporters in one hemisphere, we have demonstrated that several AEDs, including phenytoin, phenobarbital, carbamazepine, lamotrigine, and felbamate are substrates for either Pgp or MRPs or both. ${ }^{23}$ In addition, as already noted above, we use MRP2-deficient ( $\mathrm{TR}^{-}$) rats to study which AEDs are substrates for this MRP. ${ }^{50,78}$ Our experiments with phenytoin showed that rats lacking MRP2 in the BBB exhibit an enhanced brain uptake of phenytoin, which is associated with a more marked anticonvulsant effect compared to normal controls. Currently, we use different cell lines (MDCK II, LLC) transfected with either human or rodent $\mathrm{ABC}$ transporters to further characterize the interaction of AEDs with these transporters. We also demonstrated that long-term administration of AEDs, such as phenobarbital or phenytoin, in rats does not induce the expression of Pgp in the brain. ${ }^{79}$

In view of the overexpressed $\mathrm{ABC}$ transporters found in epileptogenic brain tissue of patients with pharmacoresistant epilepsy and animal models of epilepsy, another important question is whether this overexpression lowers brain uptake of AEDs. By using the kainate model of TLE in mice, Rizzi et al. ${ }^{80}$ demonstrated that the significant increase in mdr1 mRNA measured by RT-PCR in the hippocampus after kainate-induced seizures was associated with a $30 \%$ decrease in the brain/ plasma ratio of phenytoin, thus substantiating that Pgp alterations significantly affect AED concentrations in the brain.

Sisodiya $^{32}$ has recently pointed out that at least four criteria must be satisfied if we are to accept a role of a proposed drug resistance mechanism in resistant epilepsy: 1) the mechanism must be detectable in epileptogenic brain tissue; 2) the mechanism must have appropriate functionality; 3 ) the mechanism must be active in drug resistance; and 4) overcoming the mechanism should affect drug resistance. As discussed above, overexpression of $\mathrm{ABC}$ transporters has been demonstrated in epileptogenic brain tissue from patients with intractable epilepsy and animal models of epilepsy. Furthermore, as shown by animal experiments, this overexpression of multidrug transporters in the brain has the functional capacity to lower brain AED levels. Whereas these data satisfy the first and second criteria listed by Sisodiya, ${ }^{32}$ this is not sufficient to prove that overexpression of multidrug transporters is causally related to drug resistance. To address the third criterion (relevant activity of the mechanism in drug-resistant epilepsy), we used two animal models of TLE and selected AED responders and nonresponders from these models. In both models, AED nonresponders exhibited a significantly higher Pgp expression in brain capillary endothelial cells than responders. ${ }^{81,82}$ With respect to the fourth criterion of $\mathrm{Si}$ sodiya, ${ }^{32}$ i.e., demonstration that inhibition or avoidance of the resistance-mediating mechanism counteracts drug resistance in epilepsy, we have some indirect evidence from experiments with diverse AEDs in pharmaco-resistant rats selected from the kindling model of TLE. ${ }^{83}$ All AEDs that were substrates for Pgp showed absent or low anticonvulsant efficacy in nonresponders that had been selected by repeated testing with the AED phenytoin. $^{23,83}$ The only exception was levetiracetam that was as efficacious in responders and nonresponders. ${ }^{83}$ Recent data from our group showed that levetiracetam is the first AED tested in our lab that is not a substrate for Pgp. ${ }^{84}$ Thus, development of AEDs that bypass overexpressed multidrug transporters could be a valuable approach for overcoming drug resistance. For direct proof of principle, we currently examine whether Pgp inhibitors can be used to counteract multidrug resistance in drug resistant kindled rats. However, there are certainly other mechanisms of resistance in epilepsy, including epilepsy-induced changes in drug targets in the brain, ${ }^{85}$ so that the practical importance of the multidrug transporter hypothesis needs to be proven.

With respect to treatment-resistant depression, be- 
tween $29 \%$ and $46 \%$ of depressed patients fail to respond fully with antidepressant treatment of adequate dose and duration. ${ }^{60}$ The mechanisms underlying drug resistance in depression are poorly understood. Because depression and other psychoses are common comorbidities of epilepsy, particularly TLE, ${ }^{86}$ overexpression of multidrug transporters at the BBB may be involved in patients with treatment-resistant epilepsy and depression. Various antidepressants, such as amitryptiline or paroxetine, are substrates for Pgp (Table 1), and in mice lacking Pgp, penetration of these drugs into the brain is enhanced. ${ }^{87,88}$ Furthermore, several AEDs, such as carbamazepine or lamotrigine, that are Pgp substrates are also used to treat psychiatric diseases such as bipolar (manic depressive) disorders. ${ }^{89}$ Data showing that brain penetration of antidepressant drugs depend on the presence of Pgp have been proposed to offer an explanation for poor or nonresponse to antidepressant treatment. ${ }^{88}$ In addition to being substrates for Pgp, some antidepressants seem to inhibit this multidrug transporter. ${ }^{90}$ Based on these observations, Pariante et al. ${ }^{91}$ recently suggested that the known interactions between antidepressants and glucocorticoids, which are also Pgp substrates, may be mediated via Pgp. These authors proposed that antidepressants in humans could inhibit steroid transporters localized on the BBB and in neurones, like the multidrug transporter Pgp, and thus increase the access of cortisol to the brain and the glucocorticoid-mediated negative feedback on the hypothalamic-pituitary-adrenal (HPA) axis. Malfunctions of the negative feedback are considered to be a pathophysiological mechanism in the development of depression. ${ }^{92}$ Thus, enhanced cortisol action in the brain might prove to be a successful approach to maximize therapeutic antidepressant effects. ${ }^{91}$ Also, the beneficial clinical effect of the polyunsaturated $\omega$-3 fatty acid ethyl-eicosapentaenoate (EPA) when used as an adjunct in therapy-refractory depression has been related to an effect on Pgp function at the level of the BBB. ${ }^{93}$ Apart from Pgp, Shibayama et al. ${ }^{94}$ recently reported that St. John's Wort, which is widely used as a herbal medicine for the treatment of depression, markedly increases the expression of MRP2 in rats, whereas no such effect was seen for Pgp or MRP1. Considering the four criteria of Sisodiya ${ }^{32}$ described above, the evidence implicating $\mathrm{ABC}$ transporters in drug resistance in depression is currently much less convincing compared to the potential role of these efflux transporters in intractable epilepsy. Nevertheless, taken the enormous experimental and clinical interest in multidrug transporters, it seems very likely that more data on the potential role of such proteins for therapy-refractory depression will rapidly evolve.

Another example where expression of $\mathrm{ABC}$ transporters at the BBB may be associated with drug resistance are brain tumors. The efficacy of chemotherapy for ma- lignant primary or metastatic brain tumors is still poor. Because many anticancer drugs are substrates for multidrug transporters (Table 1), this is at least partly due to the presence of $A B C$ transporters such as Pgp at the BBB. ${ }^{95}$ The therapeutic importance of this problem has recently been demonstrated for the anticancer drug paclitaxel, which is a substrate for Pgp (Table 1). This cytostatic drug is of very limited use against brain tumors in patients with an intact BBB. ${ }^{14}$ When nude mice with an intracerebrally implanted human glioblastoma were pretreated with the Pgp blocker valspodar (PSC 833), brain paclitaxel concentrations increased and tumor volume markedly decreased compared with controls. ${ }^{96}$

$\mathrm{ABC}$ efflux transporters at the BBB are also involved in the resistance of brain human immunodeficiency virus (HIV) to pharmacotherapy. The brain is known to be a site of viral replication for HIV and thus is an important target tissue for antiretroviral agents. However, HIV protease inhibitors, which brought considerable progress in the treatment of HIV infection, have only limited ability to reach the CNS, with the majority of this class of drugs not detected in human CSF after administration. ${ }^{4,97}$ This seems to be partly due to the extrusion from the CNS by Pgp. ${ }^{97,98}$ Brain concentrations of indinavir, nelfinarvir, and saquinavir reach significantly increased levels in mdrla knockout mice as compared with wild-type mice. ${ }^{98-100}$ Furthermore, brain penetration of amprenavir was potentially increased by the Pgp inhibitor GF120918. ${ }^{101}$ These data demonstrated that Pgp limits the penetration of these antiviral drugs into the brain. Further proof came from investigations in an in vitro blood-brain barrier coculture model, in which polarized transport by Pgp was shown for amprenavir, ritonavir, and indinavir. ${ }^{102}$ Taken together these data indicate that Pgp-mediated efflux critically limits brain access of several HIV protease inhibitors, and thus limits virus eradication from the brain by these compounds.

In conclusion, overexpression of multidrug transporters and their genes is a novel and reasonable hypothesis to explain multidrug resistance in epilepsy and other brain diseases, but further studies are needed to establish this concept. There are certainly other mechanisms of drug resistance that need to be identified or substantiated, such as disease-related changes in drug targets. Enhanced understanding of the cellular and molecular mechanisms of drug resistance will ultimately lead to new strategies for reversal or prevention of intrinsic and acquired multidrug resistance in brain diseases such as epilepsy.

\section{ROLE OF THE BBB ABC TRANSPORTERS IN EXTRUDING DRUGS FROM THE BRAIN}

Drugs can leave the brain via the BCSFB and the $\mathrm{BBB}$. Because CSF is continuously produced, there is a 
net diffusion gradient into the CSF and a net flux out of the brain. ${ }^{103} \mathrm{CSF}$ diffuses across the arachnoid granulations, thereby carrying drugs with it into the general circulation. ${ }^{103}$ Drugs can also diffuse back across the BBB or can be actively transported from BBB endothelial cells in the blood. $\mathrm{ABC}$ transporters, which are localized in the apical membrane of BBB endothelial cells function as efflux transporters mediating an active extrusion of their substrates back into the capillary lumen. ${ }^{16}$ The transporter molecules may readily shuttle drugs back into the blood, which just entered the endothelial cell membrane or cytoplasm. ${ }^{21}$ In addition, active transport at the apical membrane generally decreases intracellular endothelial drug concentrations, and thus increases the concentration gradient between endothelial cell cytoplasm and brain extracellular space. As a consequence, the passage of drugs from the brain extracellular space into endothelial cells increases. This increase is associated with successional extrusion via the apical membrane into the blood by active transport. Furthermore, the major multidrug transporter Pgp might be involved in caveolar trafficking, and this trafficking is discussed to have a functional impact on drug transport. ${ }^{21}$

In most experimental setups, which are used to study the impact of Pgp or other ABC efflux transporters on brain distribution of drugs in vivo, it is not possible to distinguish between limitation of brain access and enhanced extrusion from the brain. Both mechanisms must be considered to coact in determining brain distribution of efflux transporter substrates. To more selectively define active drug extrusion from the brain, the drug must be administered intracerebrally, and then the impact of transporter inhibition or transporter knockout on the decline of drug concentrations in the brain must be determined. This experimental setup has not been used in the majority of studies, which investigated the impact of efflux transporters on brain pharmacokinetics of drugs.

The impact of Pgp-mediated BBB extrusion on drug efficacy is nicely illustrated by studies with opiate and opioid analgesics. Increased brain access of morphine was demonstrated in mdrla or $m d r l a / l b$ lacking mice, and was proven to be associated with an enhanced analgesic effect. ${ }^{104-106}$ In line with these data, inhibition of Pgp by cyclosporine A or by GF120918 significantly increased the brain access and the antinociceptive effect of morphine in mice or rats. ${ }^{106,107}$ Data from King et al. ${ }^{108}$ rendered new exciting insights in the functional role of Pgp efflux at the BBB. In their study, the brainto-blood transport of morphine was reduced by downregulating Pgp expression with antisense molecules. As a consequence of reduced Pgp expression morphine-induced analgesia was enhanced following systemic administration. In contrast the analgesic activity of centrally administered morphine was diminished after Pgp downregulation. This implies that a peripheral action significantly contributes to the analgesic effect of morphine, and that this is achieved by active Pgp-mediated extrusion of morphine from the brain. The efflux by Pgp proved to be not limited to drugs that act via opioid receptors, but extends to endogenous peptides, like $\beta$-endorphin, which modulate nociception via opioid receptors. ${ }^{108}$ Based on these data, it was concluded that the BBB Pgp transport system pumps functionally active compounds from the brain to the periphery, and thus may be an important regulatory mechanism for the CNS to modulate peripheral systems. ${ }^{108}$

Active transport by Pgp also contributes to brain extrusion of further opioid analgesics, like fentanyl or methadone. ${ }^{106,109}$ Thus, BBB Pgp seems to be an important issue in pain control with opioid analgesics, which may influence the onset, magnitude, and duration of the analgesic response. ${ }^{109}$ When the effect of the efflux was compared between different opioid analgesics, it proved to be more pronounced for drugs with membrane-limited cerebral kinetics, i.e., more hydrophilic drugs like morphine, as compared with drugs with flow-limited kinetics, i.e., more lipophilic drugs like fentanyl. ${ }^{110}$ This reflects the fact that the net effect of the efflux is governed by the ratio of Pgp drug clearance and the permeability of the membrane for the drug, i.e., the extent by which the drug leaks back through the membrane.

Similar to these experiments with opioids, the impact of Pgp expression at the BBB for brain penetration and extrusion has been demonstrated for various of the drugs shown in Table 1, but a detailed description of respective findings and their consequences for therapeutic efficacy and adverse effects of drugs is beyond the scope of this review. Less data are available as yet for MRPs and BCRP, but the substrate spectrum of these transporters (Table 1) suggest that they may affect brain pharmacokinetics of various drugs.

\section{HOW CAN UNDERSTANDING OF BBB ABC TRANSPORTERS BE USED TO INCREASE DRUG TRANSPORT TO THE BRAIN?}

As shown in this review, expression of $\mathrm{ABC}$ transporters at the $\mathrm{BBB}$ can potentially limit the penetration of various lipophilic drugs, including many clinically used agents, which becomes even more marked when expression is enhanced by disease states such as epilepsy. Therefore, inhibition of $\mathrm{ABC}$ transporters or bypassing these transporters can be used to enhance brain uptake of a wide variety of drugs. ${ }^{14,15}$ Such strategies to enhance drug penetration into the brain may also form a novel therapeutic approach for reversing drug resistance in brain diseases such as epilepsy. ${ }^{23}$ However, any modulation of $\mathrm{ABC}$ transporter function has to consider the potential hazards of such modulation. As shown by experiments in knockout mice, drugs that are normally well 
tolerated may become neurotoxic in the absence of $\mathrm{ABC}$ transporters at the BBB. ${ }^{6,10}$ Furthermore, it is becoming increasingly clear that multidrug transporters such as Pgp serve a variety of physiological functions apart from their role to protect cells from potentially toxic xenobiotics. For instance, recent studies have indicated that Pgp and MRPs may protect astrocytes and other cell types from apoptosis. ${ }^{11,112}$ Nevertheless, transient inhibition of ABC transporters such as Pgp by short-term administration of inhibitors may be a useful strategy to reverse or prevent pharmacoresistance due to overexpression of such transporters.

Multidrug resistance of tumor cells, whereby such cells simultaneously possess intrinsic or acquired crossresistance to diverse chemotherapeutic agents, represents a major obstacle in the successful therapy of neoplastic diseases. ${ }^{113}$ Experimentally, this resistance can be reversed by coadministration of anticancer drugs with inhibitors of Pgp or MRPs, which stimulated pharmaceutical industry to develop a large number of Pgp and MRP inhibitors (Table 1), which are in various stages of clinical development in oncology. ${ }^{113,114}$ However, modulation of Pgp in clinical oncology has had limited success as yet. ${ }^{113}$ First-generation Pgp inhibitors such as verapamil, quinidine, and cyclosporine A lacked specificity, required high doses to reverse multidrug resistance and were associated with unacceptable toxicities. ${ }^{115}$ Furthermore, several of these Pgp inhibitors led to pharmacokinetic interactions with the antineoplastic agents, resulting in reduced elimination of the anticancer agents with subsequent toxicity. Second-generation Pgp inhibitors, e.g., valspodar or biricodar, are more selective and have better tolerability but are confounded by unpredictable pharmacokinetic interactions and interactions with other transporter proteins. ${ }^{113,114}$ Furthermore, these agents may cause ataxia. Third-generation Pgp inhibitors, such as tariquidar, zosuquidar, laniquidar, and ONT-093, have high potency and specificity for Pgp. Furthermore, pharmacokinetic studies to date have shown no appreciable impact on cytochrome P450 drug metabolism and no clinically significant drug interactions with common chemotherapeutic agents. ${ }^{113,114}$ Furthermore, third-generation Pgp inhibitors have shown promise in clinical trials. The continued development of these agents may establish the true therapeutic potential of Pgp-mediated reversal of multidrug resistance in oncology and other clinical indications. In addition to Pgp inhibitors, various inhibitors of MRPs have been described (Table 1), but most of these inhibitors lack specificity and inhibit several MRPs and partly also other transporters. Vice versa, several Pgp inhibitors of the first and second generation also inhibit MRP1. Potent and specific inhibitors of MRPs and BCRP are now being developed, opening the door to clinical applications of MRP or BCRP inhibition. ${ }^{115}$ In addition to their potential use in oncology, inhibitors of multidrug transporters could also be valuable in enhancing intestinal drug absorption and increasing drug penetration through biologically important protective barriers such as the BBB. However, as pointed out above, it is uncertain whether chronic administration of such inhibitors is feasible given the protective role of these transporters in various cell types and organs.

Another potentially promising approach to enhancing the penetration of otherwise nonpermeating drugs to the brain is to by-pass $\mathrm{ABC}$ transporters at the $\mathrm{BBB}$ without direct inhibition of these transporters. ${ }^{14}$ One strategy uses antibody-coupled immunoliposomes to transport Pgp substrates. ${ }^{14,116}$ The idea is to move the encapsulated drug through the luminal plasma membrane of brain capillary endothelial cells avoiding direct interaction with Pgp or other transporters at the BBB. ${ }^{116} \mathrm{Im}$ munoliposomes, which are coupled to an antitransferrin receptor monoclonal antibody, have been shown to be internalized at the BBB by means of receptor-mediated endocytosis and to deliver Pgp substrates efficiently to the brain tissue. ${ }^{116}$ Another system makes use of drugcontaining nanoparticles, which pass through the luminal membrane of the BBB by endocytosis. ${ }^{14}$ Nanoparticles have been used to deliver several drugs into the brain and have been particularly useful in chemotherapy of disseminated and aggressive brain tumors. ${ }^{14}$

With respect to development of new drugs for treatment of brain diseases, drugs not transported by multidrug transporters expressed at the BBB would certainly lead to drugs with increased brain penetration. Furthermore, in view of the potential role of multidrug transporter overexpression at the BBB in treatment-resistant epilepsy, ${ }^{23}$ drugs not transported by multidrug transporters expressed at the BBB could have advantages toward available AEDs in patients with intractable epilepsy and focal overexpression of such transporters. Pgp assays to identify drugs that are not Pgp substrates are already routinely used in drug development in the pharmaceutical industry, ${ }^{7}$ but this does not exclude that other transporters such as MRPs or BCRP accept such drugs as substrates. However, when searching lipid-soluble drugs that are poor or no substrates for such $\mathrm{ABC}$ transporters, it should be noted that, because multidrug transporters are thought to protect a number of organs from intoxication by xenobiotics, lipophilic drugs that are not restricted in tissue distribution by such transporters may have a low therapeutic margin.

\section{CONCLUSIONS}

Because of their location at the luminal site of brain capillary endothelial cells that form the BBB, their potency as efflux pumps, and their multispecificity as drug transporters, ABC transporters such as Pgp, MRP2, and $\mathrm{BCRP}$ are an obvious target for maneuvers designed to 
modify the BBB to entry of therapeutic drugs into the brain. Furthermore, given the accumulating evidence indicating an overexpression of such $\mathrm{ABC}$ transporters at the BBB as a major pathomechanism of pharmacoresistance of brain diseases such as epilepsy, pharmacological modulation of these transporters could form a novel rational strategy to overcome medical intractability in such diseases. The availability of advanced tools such as more specific transport inhibitors, specific antibodies, knockout or transgenic animals, and in vivo imaging techniques, using positron emission tomography (PET) with ${ }^{11} \mathrm{C}$-labeled substrates will facilitate the acquisition of more detailed knowledge of the different $\mathrm{ABC}$ transporters and their interaction to modulate drug transport into the brain under physiologic and pathologic conditions.

Acknowledgments: The authors' own studies have been supported by grants (Lo 274/9-1 and /9-2) from the Deutsche Forschungsgemeinschaft (Bonn, Germany).

\section{REFERENCES}

1. Jones PM, George AM. The ABC transporter structure and mechanism: perspectives on recent research. Cell Mol Life Sci 61:682699, 2004.

2. Borges-Walmsley MI, McKeegan KS, Walmsley AR. Structure and function of efflux pumps that confer resistance to drugs. Biochem J 376:313-338, 2003.

3. Silverman JA. Multidrug-resistance transporters. Pharm Biotechnol 12:353-386, 1999.

4. Fromm MF. P-glycoprotein: a defense mechanism limiting oral bioavailability and CNS accumulation of drugs. Int J Clin Pharmacol Ther 38:69-74, 2000.

5. Litman T, Druley TE, Stein WD, Bates SE. From MDR to MXR: new understanding of multidrug resistance systems, their properties and clinical significance. Cell Mol Life Sci 58:931-959, 2001.

6. Schinkel AH, Jonker JW. Mammalian drug efflux transporters of the ATP binding cassette (ABC) family: an overview. Adv Drug Deliv Rev 55:3-29, 2003.

7. Lin JH. How significant is the role of P-glycoprotein in drug absorption and brain uptake? Drugs Today (Barc) 40:5-22, 2004.

8. Fromm MF. Importance of P-glycoprotein for drug disposition in humans. Eur J Clin Invest 33[Suppl 2]:6-9, 2003.

9. Sun H, Dai H, Shaik N, Elmquist WF. Drug efflux transporters in the CNS. Adv Drug Deliv Rev 55:83-105, 2003.

10. Schinkel AH. P-Glycoprotein, a gatekeeper in the blood-brain barrier. Adv Drug Deliv Rev 36:179-194, 1999.

11. Pardridge WM. Blood-brain barrier biology and methodology. J Neurovirol 5:556-569, 1999.

12. Graff CL, Pollack GM. Drug transport at the blood-brain barrier and the choroid plexus. Curr Drug Metab 5:95-108, 2004.

13. Gerk PM, Vore M. Regulation of expression of the multidrug resistance-associated protein 2 (MRP2) and its role in drug disposition. J Pharmacol Exp Ther 302:407-415, 2002.

14. Fricker G, Miller DS. Modulation of drug transporters at the blood-brain barrier. Pharmacology 70:169-176, 2004.

15. Begley DJ. ABC transporters and the blood-brain barrier. Curr Pharm Des 10:1295-1312, 2004.

16. Lee G, Dallas S, Hong M, Bendayan R. Drug transporters in the central nervous system: brain barriers and brain parenchyma considerations. Pharmacol Rev 53:569-596, 2001.

17. Golden PL, Pollack GM. Blood-brain barrier efflux transport. J Pharm Sci 92:1739-1753, 2003.

18. Juliano RL, Ling V. A surface glycoprotein modulating drug permeability in Chinese hamster ovary cell mutants. Biochim Biophys Acta 455:152-162, 1976.
19. Cordon-Cardo C, O'Brien JP, Casals D, Rittman-Grauer L, Biedler JL, Melamed MR, Bertino JR. Multidrug-resistance gene (P-glycoprotein) is expressed by endothelial cells at blood-brain barrier sites. Proc Natl Acad Sci USA 86:695-698, 1989.

20. Thiebaut F, Tsuruo T, Hamada H, Gottesman MM, Pastan I, Willingham MC. Immunohistochemical localization in normal tissues of different epitopes in the multidrug transport protein P170: evidence for localization in brain capillaries and crossreactivity of one antibody with a muscle protein. $J$ Histochem Cytochem 37:159-164, 1989.

21. Demeule M, Regina A, Jodoin J, Laplante A, Dagenais C, Berthelet F, Moghrabi A, Beliveau R. Drug transport to the brain: key roles for the efflux pump P-glycoprotein in the blood-brain barrier. Vascul Pharmacol 38:339-348, 2002.

22. Sawchuk RJ, Elmquist WF. Microdialysis in the study of drug transporters in the CNS. Adv Drug Deliv Rev 45:295-307, 2000.

23. Löscher W, Potschka H. Role of multidrug transporters in pharmacoresistance to antiepileptic drugs. J Pharmacol Exp Ther 301:7-14, 2002.

24. Pardridge WM, Golden PL, Kang YS, Bickel U. Brain microvascular and astrocyte localization of P-glycoprotein. J Neurochem 68:1278-1285, 1997.

25. Golden PL, Pardridge WM. P-Glycoprotein on astrocyte foot processes of unfixed isolated human brain capillaries. Brain Res 819:143-146, 1999.

26. Golden PL, Pardridge WM. Brain microvascular P-glycoprotein and a revised model of multidrug resistance in brain. Cell Mol Neurobiol 20:165-181, 2000.

27. Abbott NJ, Khan EU, Rollinson CMS, Reichel A, Janigro D, Dombrowski SM, Dobbie MS, Begley DJ. Drug resistance in epilepsy: the role of the blood-brain barrier. In: Mechanisms of drug resistance in epilepsy. Lessons from oncology (Ling V, ed), pp 38-47. Chichester: Wiley, Ltd., 2002.

28. O'Brien JP, Cordon-Cardo C. P-glycoprotein expression in normal human tissues. In: Multidrug resistance in cancer cellls (Gupta S, Tsuruo T, eds), pp 285-291. Chichester: Wiley, Ltd., 1996.

29. Scheffer GL, Scheper RJ. Drug resistance molecules: lessons from oncology. Novartis Found Symp 243:19-31, 2002.

30. Schlachetzki F, Pardridge WM. P-glycoprotein and caveolin-1alpha in endothelium and astrocytes of primate brain. Neuroreport 14:2041-2046, 2003.

31. Marroni M, Marchi N, Cucullo L, Abbott NJ, Signorelli K, Janigro D. Vascular and parenchymal mechanisms in multiple drug resistance: a lesson from human epilepsy. Curr Drug Targets 4:297-304, 2003.

32. Sisodiya SM. Mechanisms of antiepileptic drug resistance. Curr Opin Neurol 16:197-201, 2003.

32a. Volk H, Potschka H, Löscher W. Immunohistochemical localization of p-glycoprotein in rat brain and detection of its increased expression by seizures are sensitive to fixation and staining variables. J Histochem Cytochem, in press.

33. Volk HA, Burkhardt K, Potschka H, Chen J, Becker A, Löscher W. Neuronal expression of the drug efflux transporter P-glycoprotein in the rat hippocampus after limbic seizures. Neuroscience 123:751-759, 2004.

34. Borst P, Evers R, Kool M, Wijnholds J. A family of drug transporters: the multidrug resistance-associated proteins. J Natl Cancer Inst 92:1295-1302, 2000.

35. Seelig A, Blatter XL, Wohnsland F. Substrate recognition by P-glycoprotein and the multidrug resistance-associated protein MRP1: a comparison. Int J Clin Pharmacol Ther 38:111-121, 2000.

36. Borst P, Evers R, Kool M, Wijnholds J The multidrug resistance protein family. Biochim Biophys Acta 1461:347-357, 1999.

37. Zhang Y, Han H, Elmquist WF, Miller DW. Expression of various multidrug resistance-associated protein (MRP) homologues in brain microvessel endothelial cells. Brain Res 876:148-153, 2000.

38. Miller DS, Nobmann SN, Gutmann H, Toeroek M, Drewe J, Fricker G. Xenobiotic transport across isolated brain microvessels studied by confocal microscopy. Mol Pharmacol 58:1357-1367, 2000. 
39. Dombrowski SM, Desai SY, Marroni M, Cucullo L, Goodrich K, Bingaman W, Mayberg MR, Bengez L, Janigro D. Overexpression of multiple drug resistance genes in endothelial cells from patients with refractory epilepsy. Epilepsia 42:1501-1506, 2001.

40. Decleves X, Regina A, Laplanche JL, Roux F, Boval B, Launay JM, Scherrmann JM. Functional expression of P-glycoprotein and multidrug resistance-associated protein (Mrp1) in primary cultures of rat astrocytes. J Neurosci Res 60:594-602, 2000.

41. Rao VV, Dahlheimer JL, Bardgett ME, Snyder AZ, Finch RA, Sartorelli AC, Piwnica-Worms D. Choroid plexus epithelial expression of MDR1 P glycoprotein and multidrug resistance-associated protein contribute to the blood-cerebrospinal-fluid drugpermeability barrier. Proc Natl Acad Sci USA 96:3900-3905, 1999.

42. Choudhuri S, Cherrington NJ, Li N, Klaassen CD. Constitutive expression of various xenobiotic and endobiotic transporter mRNAs in the choroid plexus of rats. Drug Metab Dispos 31:1337$1345,2003$.

43. Hirrlinger J, Konig J, Dringen R. Expression of mRNAs of multidrug resistance proteins (Mrps) in cultured rat astrocytes, oligodendrocytes, microglial cells and neurones. J Neurochem 82: 716-719, 2002.

44. Sun H, Johnson DR, Finch RA, Sartorelli AC, Miller DW, Elmquist WF. Transport of fluorescein in MDCKII-MRP1 transfected cells and mrp1-knockout mice. Biochem Biophys Res Commun 284:863-869, 2001.

45. Wijnholds J, deLange EC, Scheffer GL, van den Berg DJ, Mol CA, van der Valk M, Schinkel AH, Scheper RJ, Breimer DD, Borst P. Multidrug resistance protein 1 protects the choroid plexus epithelium and contributes to the blood-cerebrospinal fluid barrier. J Clin Invest 105:279-285, 2000.

46. Koopen NR, Wolters H, Havinga R, Vonk RJ, Jansen PL, Muller M, Kuipers F. Impaired activity of the bile canalicular organic anion transporter (Mrp2/cmoat) is not the main cause of ethinylestradiol-induced cholestasis in the rat. Hepatology 27:537545,1998

47. Gutmann H, Torok M, Fricker G, Huwyler J, Beglinger C, Drewe J. Modulation of multidrug resistance protein expression in porcine brain capillary endothelial cells in vitro. Drug Metab Dispos 27:937-941, 1999.

48. Potschka H, Löscher W. Multidrug resistance-associated protein is involved in the regulation of extracellular levels of phenytoin in the brain. Neuroreport 12:2387-2389, 2001.

49. Potschka H, Fedrowitz M, Löscher W. P-glycoprotein and multidrug resistance-associated protein are involved in the regulation of extracellular levels of the major antiepileptic drug carbamazepine in the brain. Neuroreport 12:3557-3560, 2001.

50. Potschka H, Fedrowitz M, Löscher W. Multidrug resistance protein MRP2 contributes to blood-brain barrier function and restricts antiepileptic drug activity. J Pharmacol Exp Ther 306: 124-131, 2003.

51. Eisenblatter T, Huwel S, Galla HJ. Characterisation of the brain multidrug resistance protein (BMDP/ABCG2/BCRP) expressed at the blood-brain barrier. Brain Res 971:221-231, 2003.

52. Cisternino S, Mercier C, Bourasset F, Roux F, Scherrmann JM. Expression, up-regulation, and transport activity of the multidrugresistance protein Abcg2 at the mouse blood-brain barrier. Cancer Res 64:3296-3301, 2004.

53. Cooray HC, Blackmore CG, Maskell L, Barrand MA. Localisation of breast cancer resistance protein in microvessel endothelium of human brain. Neuroreport 13:2059-2063, 2002.

54. Seelig A. How does P-glycoprotein recognize its substrates? Int J Clin Pharmacol Ther 36:50-54, 1998.

55. Neyfakh AA. Mystery of multidrug transporters: the answer can be simple. Med Microbiol 44:1123-1130, 2002.

56. Stouch TR, Gudmundsson O. Progress in understanding the structure-activity relationships of P-glycoprotein. Adv Drug Deliv Rev 54:315-328, 2002.

57. Haimeur A, Conseil G, Deeley RG, Cole SP. The MRP-related and BCRP/ABCG2 multidrug resistance proteins: biology, substrate specificity and regulation. Curr Drug Metab 5:21-53, 2004.

58. Löscher W. Current status and future directions in the pharmacotherapy of epilepsy. Trends Pharmacol Sci 23:113-118, 2002.
59. Jansen G, Scheper RJ, Dijkmans BA. Multidrug resistance proteins in rheumatoid arthritis, role in disease-modifying antirheumatic drug efficacy and inflammatory processes: an overview. Scand J Rheumatol 32:325-336, 2003.

60. Fava M, Davidson KG. Definition and epidemiology of treatment-resistant depression. Psychiatr Clin North Am 19:179-200, 1996.

61. Hellewell JS. Treatment-resistant schizophrenia: reviewing the options and identifying the way forward. J Clin Psychiatry 60[Suppl 23]:14-19, 1999.

62. Kwan P, Brodie MJ. Early identification of refractory epilepsy. N Engl J Med 342:314-319, 2000.

63. Tishler DM, Weinberg KT, Hinton DR, Barbaro N, Annett GM, Raffel C. MDR1 gene expression in brain of patients with medically intractable epilepsy. Epilepsia 36:1-6, 1995.

64. Duncan R, Todd N. Epilepsy and the blood-brain barrier. $\mathrm{Br} J$ Hosp Med 45:32-34, 1991.

65. Sisodiya SM, Lin WR, Harding BN, Squier MV, Thom M. Drug resistance in epilepsy: expression of drug resistance proteins in common causes of refractory epilepsy. Brain 125:22-31, 2002.

66. Sisodiya SM, Heffernan J, Squier MV. Over-expression of Pglycoprotein in malformations of cortical development. Neuroreport 10:3437-3441, 1999.

67. Aronica E, Gorter JA, Jansen GH, van Veelen CW, van Rijen PC, Leenstra S, Ramkema M, Scheffer GL, Scheper RJ, Troost D. Expression and cellular distribution of multidrug transporter proteins in two major causes of medically intractable epilepsy: focal cortical dysplasia and glioneuronal tumors. Neuroscience 118: 417-429, 2003

68. Aronica E, Gorter JA, Ramkema M, Redeker S, Ozbas-Gercerer F, van Vliet EA, Scheffer GL, Scheper RJ, van d, V, Baayen JC, Troost D. Expression and cellular distribution of multidrug resistance-related proteins in the hippocampus of patients with mesial temporal lobe epilepsy. Epilepsia 45:441-451, 2004.

69. Zhang L, Ong WY, Lee T. Induction of P-glycoprotein expression in astrocytes following intracerebroventricular kainate injections. Exp Brain Res 126:509-516, 1999.

70. Seegers U, Potschka H, Löscher W. Transient increase of Pglycoprotein expression in endothelium and parenchyma of limbic brain regions in the kainate model of temporal lobe epilepsy. Epilepsy Res 51:257-268, 2002.

71. Volk HA, Potschka H, Löscher W. Increased expression of the multidrug transporter P-glycoprotein in limbic brain regions after amygdala-kindled seizures in rats. Epilepsy Res 58:67-79, 2004.

72. Sisodiya SM, Lin WR, Squier MV, Thom M. Multidrug-resistance protein 1 in focal cortical dysplasia. Lancet 357:42-43, 2001.

73. Sisodiya SM, Martinian L, Scheffer GL, van der Valk D, Cross JH, Scheper RJ, Harding BN, Thom M. Major vault protein, a marker of drug resistance, is upregulated in refractory epilepsy. Epilepsia 44:1388-1396, 2003.

74. Regesta G, Tanganelli P. Clinical aspects and biological bases of drug-resistant epilepsies. Epilepsy Res 34:109-122, 1999.

75. Kerb R, Hoffmeyer S, Brinkmann U. ABC drug transporters: hereditary polymorphisms and pharmacological impact in MDR1, MRP1 and MRP2. Pharmacogenomics 2:51-64, 2001.

76. Siddiqui A, Kerb R, Weale ME, Brinkmann U, Smith A, Goldstein DB, Wood NW, Sisodiya SM. Association of multidrug resistance in epilepsy with a polymorphism in the drug-transporter gene ABCB1. N Engl J Med 348:1442-1448, 2003.

77. Lazarowski A, Sevlever G, Taratuto A, Massaro M, Rabinowicz A. Tuberous sclerosis associated with MDR1 gene expression and drug- resistant epilepsy. Pediatr Neurol 21:731-734, 1999.

78. Potschka H, Fedrowitz M, Löscher W. Brain access and anticonvulsant efficacy of carbamazepine, lamotrigine, and felbamate in ABCC2/MRP2-deficient TR - rats. Epilepsia 44:1479-1486, 2003.

79. Seegers U, Potschka H, Löscher W. Lack of effects of prolonged treatment with phenobarbital or phenytoin on the expression of $\mathrm{P}$-glycoprotein in various rat brain regions. Eur $J$ Pharmacol 451:149-155, 2002.

80. Rizzi M, Caccia S, Guiso G, Richichi C, Gorter JA, Aronica E, Aliprandi M, Bagnati R, Fanelli R, D’Incalci M, Samanin R, 
Vezzani A. Limbic seizures induce P-glycoprotein in rodent brain: functional implications for pharmacoresistance. J Neurosci 22:5833-5839, 2002.

81. Löscher W, Volk H, Brandt C, Potschka H. The multidrug transporter P-glycoprotein is overexpressed in limbic brain regions of pharmacoresistant epileptic rats. Program No. 906.2, 2004 Abstract Viewer/Itinerary Planner. Washington, DC: Society for Neuroscience, 2004. Online.

82. Potschka H, Volk HA, Löscher W. Pharmacoresistance and expression of multidrug transporter P-glycoprotein in kindled rats. Neuroreport 15:1657-1661, 2004.

83. Löscher W. Animal models of drug-resistant epilepsy. Novartis Found Symp 243:149-159; discussion 159-166, 2002.

84. Potschka H, Baltes S, Löscher W. Inhibition of multidrug transporters by verapamil or probenecid does not alter blood-brain barrier penetration of levetiracetam in rats. Epilepsy Res 58:8591, 2004.

85. Remy S, Gabriel S, Urban BW, Dietrich D, Lehmann TN, Elger $\mathrm{CE}$, Heinemann U, Beck H. A novel mechanism underlying drug resistance in chronic epilepsy. Ann Neurol 53:469-479, 2003.

86. Gilliam F, Hecimovic H, Sheline Y. Psychiatric comorbidity, health, and function in epilepsy. Epilepsy Behav Suppl 4:S26S30, 2003.

87. Uhr M, Steckler T, Yassouridis A, Holsboer F. Penetration of amitriptyline, but not of fluoxetine, into brain is enhanced in mice with blood-brain barrier deficiency due to mdrla P-glycoprotein gene disruption. Neuropsychopharmacology 22:380-387, 2000.

88. Uhr M, Grauer MT, Holsboer F. Differential enhancement of antidepressant penetration into the brain in mice with abcb1ab (mdrlab) P-glycoprotein gene disruption. Biol Psychiatry 54: 840-846, 2003.

89. Rogawski MA, Löscher W. The neurobiology of antiepileptic drugs for the treatment of nonepileptic conditions. Nat Med 10: 685-692, 2004.

90. Weiss J, Dormann SM, Martin-Facklam M, Kerpen CJ, KetabiKiyanvash N, Haefeli WE. Inhibition of P-glycoprotein by newer antidepressants. J Pharmacol Exp Ther 305:197-204, 2003.

91. Pariante CM, Thomas SA, Lovestone S, Makoff A, Kerwin RW. Do antidepressants regulate how cortisol affects the brain? Psychoneuroendocrinology 29:423-447, 2004.

92. Plotsky PM, Owens MJ, Nemeroff CB. Psychoneuroendocrinology of depression. Hypothalamic-pituitary-adrenal axis. Psychiatr Clin North Am 21:293-307, 1998.

93. Murck H, Song C, Horrobin DF, Uhr M. Ethyl-eicosapentaenoate and dexamethasone resistance in therapy-refractory depression. Int J Neuropsychopharmacol 7:341-349, 2004.

94. Shibayama Y, Ikeda R, Motoya T, Yamada K. St. John's Wort (Hypericum perforatum) induces overexpression of multidrug resistance protein 2 (MRP2) in rats: a 30-day ingestion study. Food Chem Toxicol 42:995-1002, 2004.

95. Bart J, Groen HJ, Hendrikse NH, van der Graaf WT, Vaalburg W, de Vries EG. The blood-brain barrier and oncology: new insights into function and modulation. Cancer Treat Rev 26:449-462, 2000.

96. Fellner S, Bauer B, Miller DS, Schaffrik M, Fankhanel M, Spruss T, Bernhardt G, Graeff C, Farber L, Gschaidmeier H, Buschauer A, Fricker G. Transport of paclitaxel (Taxol) across the bloodbrain barrier in vitro and in vivo. J Clin Invest 110:1309-1318, 2002.

97. Thomas SA. Anti-HIV drug distribution to the central nervous system. Curr Pharm Des 10:1313-1324, 2004.

98. Kim RB. Drug transporters in HIV therapy. Top HIV Med 11: 136-139, 2003.

99. Kim RB, Fromm MF, Wandel C, Leake B, Wood AJ, Roden DM, Wilkinson GR. The drug transporter P-glycoprotein limits oral absorption and brain entry of HIV-1 protease inhibitors. $J$ Clin Invest 101:289-294, 1998.

100. Washington CB, Wiltshire HR, Man M, Moy T, Harris SR, Worth E, Weigl P, Liang Z, Hall D, Marriott L, Blaschke TF. The disposition of saquinavir in normal and P-glycoprotein deficient mice, rats, and in cultured cells. Drug Metab Dispos 28:1058$1062,2000$.

101. Edwards JE, Brouwer KR, McNamara PJ. GF120918, a P-glycoprotein modulator, increases the concentration of unbound amprenavir in the central nervous system in rats. Antimicrob Agents Chemother 46:2284-2286, 2002.

102. van der Sandt I, Vos CM, Nabulsi L, Blom-Roosemalen MC, Voorwinden HH, de Boer AG, Breimer DD. Assessment of active transport of HIV protease inhibitors in various cell lines and the in vitro blood-brain barrier. AIDS 15:483-491, 2001.

103. Carvey PM. The delivery of drugs to the CNS: Systemic delivery with central action. In: Drug action in the central nervous system (Carvey PM, ed), pp 34-42. New York: Oxford University Press, 1998.

104. Xie R, Hammarlund-Udenaes M, de Boer AG, de Lange EC. The role of P-glycoprotein in blood-brain barrier transport of morphine: transcortical microdialysis studies in mdr1a $(-/-)$ and mdr1a (+/+) mice. Br J Pharmacol 128:563-568, 1999.

105. Zong J, Pollack GM. Morphine antinociception is enhanced in mdr1a gene-deficient mice. Pharm Res 17:749-753, 2000.

106. Thompson SJ, Koszdin K, Bernards CM. Opiate-induced analgesia is increased and prolonged in mice lacking P-glycoprotein. Anesthesiology 92:1392-1399, 2000.

107. Letrent SP, Pollack GM, Brouwer KR, Brouwer KL. Effects of a potent and specific P-glycoprotein inhibitor on the blood-brain barrier distribution and antinociceptive effect of morphine in the rat. Drug Metab Dispos 27:827-834, 1999.

108. King M, Su W, Chang A, Zuckerman A, Pasternak GW. Transport of opioids from the brain to the periphery by P-glycoprotein: peripheral actions of central drugs. Nat Neurosci 4:268-274, 2001.

109. Dagenais C, Graff CL, Pollack GM. Variable modulation of opioid brain uptake by P-glycoprotein in mice. Biochem Pharmacol 67:269-276, 2004.

110. Upton RN. Theoretical aspects of P-glycoprotein mediated drug efflux on the distribution volume of anaesthetic-related drugs in the brain. Anaesth Intensive Care 30:183-191, 2002.

111. Pallis M, Turzanski J, Higashi Y, Russell N. P-glycoprotein in acute myeloid leukaemia: therapeutic implications of its association with both a multidrug-resistant and an apoptosis-resistant phenotype. Leuk Lymphoma 43:1221-1228, 2002.

112. Gennuso F, Fernetti C, Tirolo C, Testa N, L'Episcopo F, Caniglia S, Morale MC, Ostrow JD, Pascolo L, Tiribelli C, Marchetti B. Bilirubin protects astrocytes from its own toxicity by inducing up-regulation and translocation of multidrug resistance-associated protein 1 (Mrp1). Proc Natl Acad Sci USA 101:2470-2475, 2004.

113. Bates SF, Chen C, Robey R, Kang M, Figg WD, Fojo T. Reversal of multidrug resistance: lessons from clinical oncology. Novartis Found Symp 243:83-96, 2002.

114. Thomas H, Coley HM. Overcoming multidrug resistance in cancer: an update on the clinical strategy of inhibiting p-glycoprotein. Cancer Control 10:159-165, 2003.

115. Doyle LA, Ross DD. Multidrug resistance mediated by the breast cancer resistance protein BCRP (ABCG2). Oncogene 22:73407358, 2003.

115. Tan B, Piwnica-Worms D, Ratner L. Multidrug resistance transporters and modulation. Curr Opin Oncol 12:450-458, 2000.

116. Huwyler J, Cerletti A, Fricker G, Eberle AN, Drewe J By-passing of P-glycoprotein using immunoliposomes. J Drug Target 10:7379, 2002. 